\title{
Electron Holography in Gaseous and Liquid Environment
}

Jes Aerøe Hyllested, G. Prabhu Sai Balasubramanian, Elisabetta Maria Fiordaliso, Murat Yesibolati, Kristian Mølhave and Marco Beleggia

Technical University of Denmark, Kgs. Lynngby, Hovedstaden, Denmark

Carrying out electron holography $(\mathrm{EH})$ in gaseous and liquid environment offers a whole new range of opportunities to study in-situ physical and chemical reactions of materials, liquid-solid interfaces, plasma effects, and beam effects in general. Inelastic interactions between beam electrons and the molecules of the gas or liquid produce ions, radicals, molecular fragments, free electrons and protons, and many other reactive species that promote local chemical/physical transformations of the sample, whose effects can be directly observed with the phase-sensitive eye of EH.

To establish gas-EH in an environmental TEM (ETEM), inspire by the work of Duchamp et al. [1] we have designed and fabricated an exchangeable electron biprism, which is resistant to corrosive gasses and placed it within the ETEM column. We have assessed its performance with various gasses, $\mathrm{H}_{2}, \mathrm{~N}_{2}, \mathrm{O}_{2}$, by measuring the change in overlap width, visibility, electron count and phase sensitivity as a function of pressure. The analysis of the data shed light into fundamental aspects of gas-electron interactions, especially regarding coherence coherence loss. Our findings reveal that gas-EH is a viable setup that opens the door to a whole new class of experiments, within fields such as magnetic nanoparticles, catalysis and doped semiconductors. We have then used gas-EH to follow the progressive oxidation of $\mathrm{FeO}$ nanoparticles from magnetite to maghemite to hematite, and the results of these experiments will be presented at the conference.

In another experiment, we have deposited a carbon layer from a gas precursor on the shell of a core-shell structured GaAs nanowire with undoped core and C-doped $p$ shell, i.e. a radial $i$ - $p$ junction. Then we obtained off-axis electron holograms as a function of in-vacuum annealing temperature in the range 300$700{ }^{\circ} \mathrm{C}$ inside a TEM. For temperatures of $400{ }^{\circ} \mathrm{C}$ and above we observed changes in the electrostatic potential profile across the nanowire, likely caused by the variation in the $\mathrm{C}$ concentration profile across the nanowire caused by the combined effects of electron irradiation and annealing. In the sample we analyzed, we observed the core to be slightly off-centered, and we measured different potential steps for the $i$ - $p$ junctions on opposite sides of the core. We interpret this as a full depletion of the $i$ channel on the narrower side of the core.

The combination of EH with nanochannel liquid-cell [2] enables quantitative measurement of the mean inner potential (MIP) of liquids as a function of their molecular weight and ionicity. Understanding the MIP of liquid will help improve our experimental and theoretical knowledge of electric potentials at liquid-solid boundaries, and it is essential to achieve a deeper understanding of the driving forces behind interfacial processes [3]. Liquid-EH also allows us to probe the charge state of nanoparticles directly in liquid solution at single-particle level, based on a Laplacian transformation of the phase image reconstructed from holograms [4,5], and will lead to a deeper understanding of the fundamental processes triggered by electrostatic interactions in liquids. 

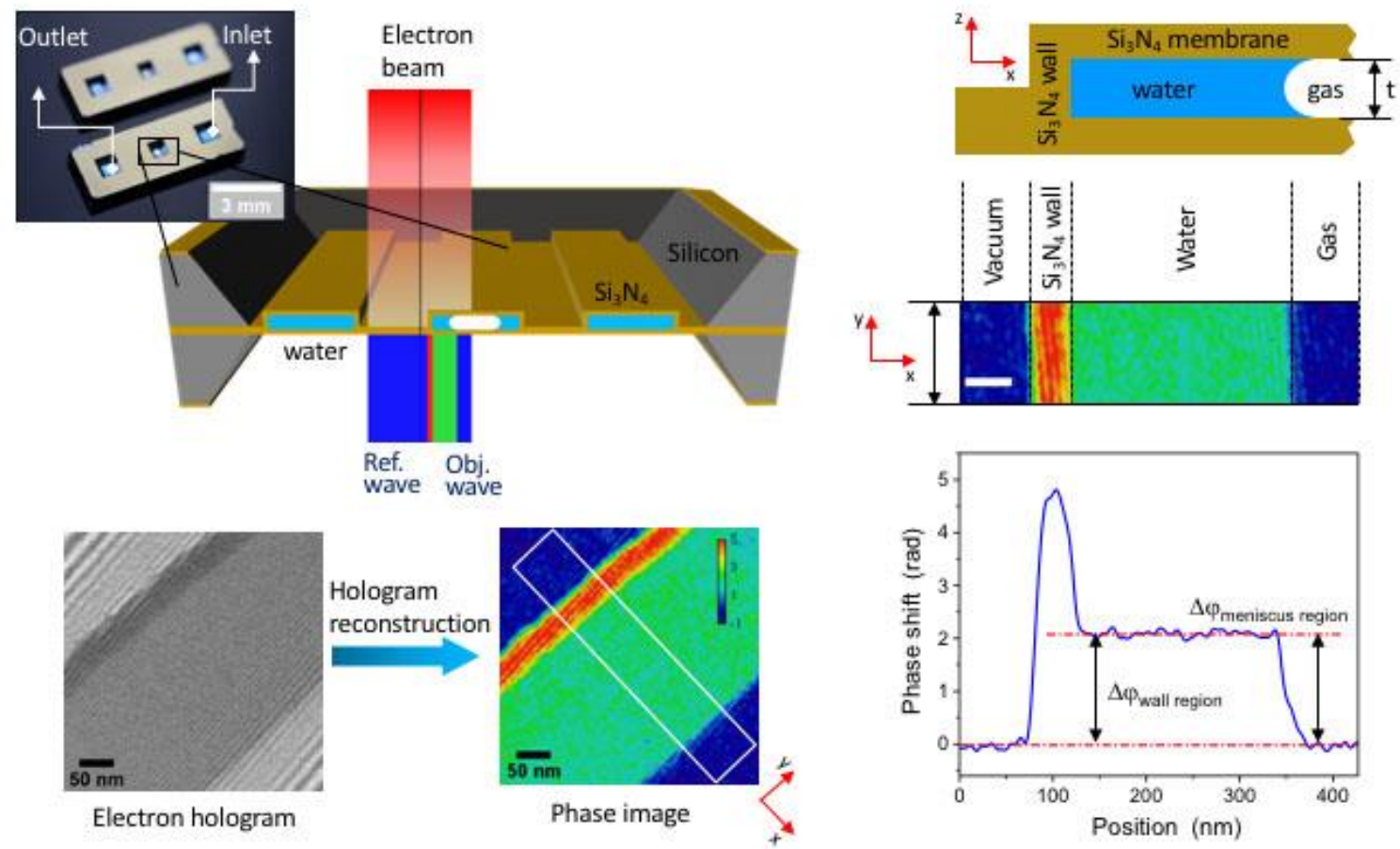

Figure 1. Top left: overview of the custom-made nanochannel liquid-cell designed to be compatible with electron holography. Bottom left: an example hologram an corresponding reconstructed phase image. Right column: correspondence between features/areas of the nanochannel, phase image, and phase profile across the region.

\section{References}

[1] M. Duchamp et al., Ultramicroscopy 185 (2018) 81

[2] S. Laganá, et al., Microelectron. Eng. 176 (2017) 71

[3] M.N. Yesibolati et al., Phys. Rev. Lett. 124 (2020) 065502

[4] M. Beleggia et al., J. Phys. D: Appl. Phys. 49 (2016) 294003

[5] M. Beleggia et al., Appl. Phys. Lett. 98 (2011) 243101 